\title{
Review on Robotic Machine to Solve the Matrix with Natural Language Processing and Image Processing
}

\author{
Prof. Sonali Zunke ${ }^{1}$, Sandesh Ukey ${ }^{2}$, Dipak Mendhe ${ }^{2}$ \\ ${ }^{1}$ Professor in CSE-IT Department, JD College of Engineering and Management, Nagpur, Maharashtra, India \\ ${ }^{2}$ Student, CSE-IT Department, JD College of Engineering and Management, Nagpur, Maharashtra, India
}

\section{Article Info \\ Volume 7, Issue 3 \\ Page Number: 395-402}

\section{Publication Issue :}

May-June-2021

\section{Article History}

Accepted : 22 May 2021

Published : 31 May 2021

\section{ABSTRACT}

As the world is moving faster, humans are not taking a step back to make the world more better place, may it be by enhanced technology or extreme creativity. We know that a human life now is full of technology and every little thing is just a click away that is the favour of automation for everything. If we explore Automation more, the major concepts which will still the spotlight are Artificial Intelligent (AI), Machine Learning (ML) and the list will continue. The other concept which steals the limelight and make every automation possible is the programming language we use to make the features we are now using possible. The language which is exponentially gaining fame is Python. If all the above mentioned technology is combined together, we get most of the automation possible today. At every corner of the world, this technology is being used to make things lively and possible. Not only companies but every industry (food, entertainment, education, manufacturers and many more) are relying on this technology. The calculation which plays a significant role in almost every bit is also being automated to make the things very simpler and quicker. Even though, we have calculator but it still cannot solve the problems which are needed to be solve in some platforms. In this paper, we shall be discussing about a similar concept.

Keywords : Artificial Intelligence (AI), Machine Learning (ML), Image processing, Raspberry Pi, Python.

\section{INTRODUCTION}

As the world advances further in the whole new era of automation, everyone is getting familiar and more reliant on it. The things which used to be hectic and time consuming are now being converted into easier and quick alternatives. The technology which is helping achieve the required goal efficiently uses concepts of Artificial Intelligence, Machine Learning, Data science, Image Processing, Deep Learning, etc combined with the programming language like python, Java, and so one. Every machine which is getting the task done is built on coding and the above mentioned technologies. Some of them use their basis

Copyright: (C) the author(s), publisher and licensee Technoscience Academy. This is an open-access article distributed under the terms of the Creative Commons Attribution Non-Commercial License, which permits unrestricted non-commercial use, distribution, and reproduction in any medium, provided the original work is properly cited 
as mathematics and this plays a significant role. The very crucial step which is required for each is the part of calculation. Even though the calculator is invented but still it can't solve the higher mathematical equations. For example, Image processing uses mathematics as the basic and they are Histogram Equalization, Probability and Statistics, Discrete Cosine Transforms, Fourier Transforms, Differential Equations, Integration, Matrix and Algebra. It is indeed not very unchallenging to solve this equations in one blow without getting errors. Unless and until it is calculated by machine, the process of solving is a bit lengthy and inconvenient. The saying goes very well that mathematics helps us prevent chaos and create orderly life. Thus, we always need an effortless and errorless methods for it. In this method we are discussing the device which will work as a calculator and will solve lengthy errorless mathematical problems. As this device will be help solve problems, the features which will make it more warm is the ability of the device to give the audio response. The audio response will make sure that the device is working, this will not only give us the idea that it is working but will also ease us. The audio response will read out the question given to solve as well as the answer the machine solved. This device which we are discussing now will basically do two things, and they are solving the higher mathematical equations and reading out the question inputted to solve as well as calculated answer. To make it easier for the user, the device will use the scanner to scan the question and read out the question once scanned fully. The device will take its standard time to calculate the Mathematical equation and later read out the answer as well as display on the device.

\section{LITERATURE REVIEW}

\section{A. Raspberry Pi}

Raspberry $\mathrm{Pi}$ is using the Advanced Reduced Instruction Set Computing Machine (ARM) technology. ARM technology is used on the board which reduces cost, heat and power consumption. It is energy effective multi core CPU implemented as System-On-Chip (SoC) weighing $50 \mathrm{gm}$ and operates on $5 \mathrm{~V}, 700 \mathrm{~mA}$ power rating. This board is available in three models named A, B, B+. The B+ Raspberry Pi board is the latest version among them, and it runs on ARM11 processor with 512MB RAM operating at 700 $\mathrm{MHz}$ frequency. It has SD card slot, which is used for booting the operating systems like Raspbian, Pidora, Raspbm. It has four USB2.0 ports to connect to the peripherals like mouse, keyboard and Wi-Fi adapter etc, making it as a full sized portable pocket computer. It also has an Ethernet port to connect to the network. GPIO ports are used to interface and control the LED's, switches, sensors and other devices. With the help of HDMI port, all kinds of monitors like LCD screens, projectors, TVs can be connected. In this board, some additional features like camera connector is available to interface camera and an audio jack. With all these features, Raspberry $\mathrm{Pi}$ is not just limited to single use, it can be used in many applications.

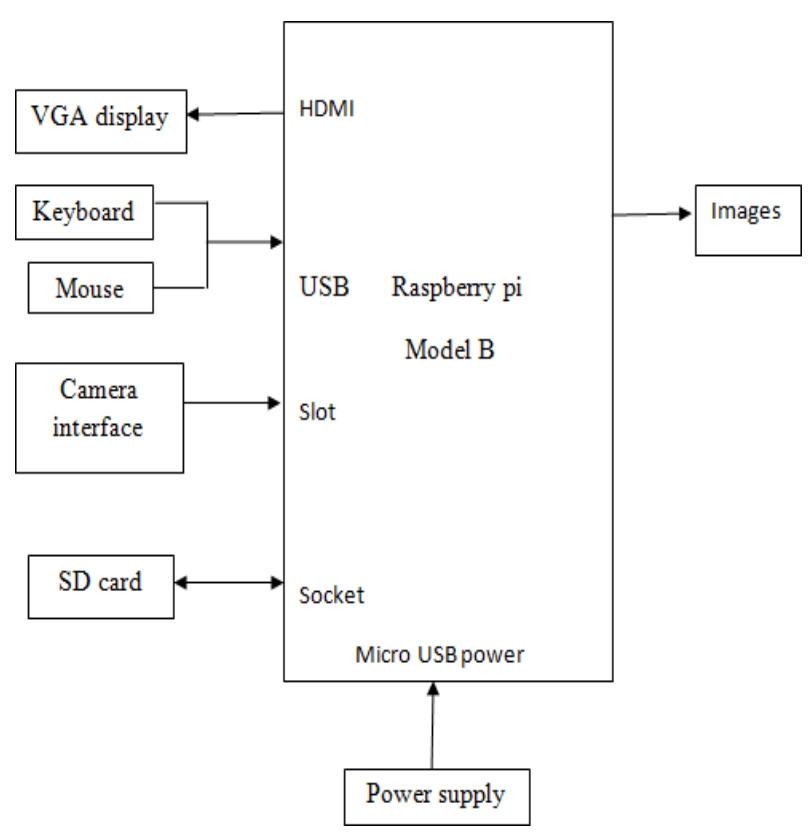

Fig 1: Image processing on Raspberry Pi 


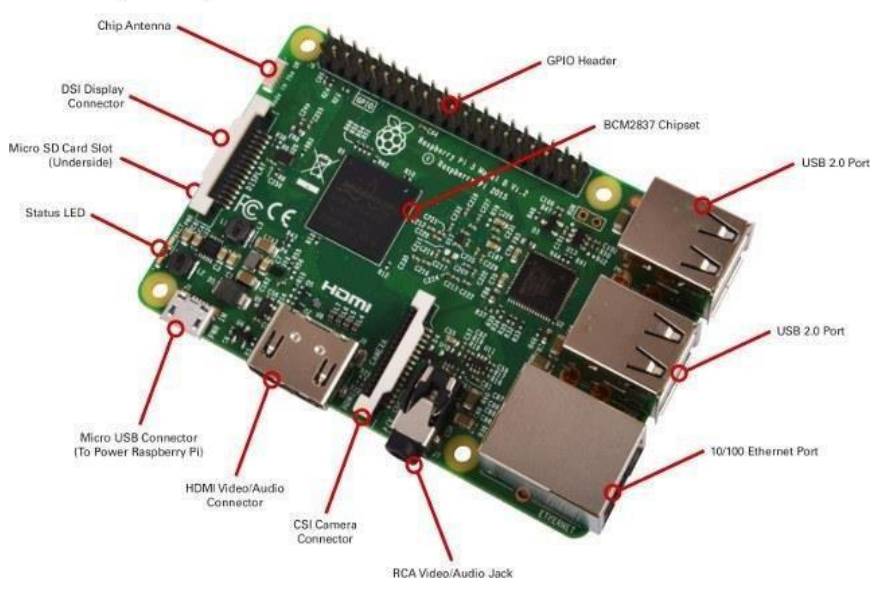

Fig 2: Raspberry pi 3 components

\section{B. Raspberry Pi Camera}

The Raspi camera plays a key role in the designed architecture, as it is used to capture images and also used for recording videos. The camera supports the latest rasbian version and operating system of the raspberry pi. It used to capture images and high definition (HD) Video [15]. It supports all models of raspberry pi. It is applicable in home security and camera traps of wildlife. It supports different modes such VGA90, 720p60, 1080p30. The raspberry pi camera module is shown in fig 4 .

The Camera Module can be used to take highdefinition video, as well as stills photographs. It's easy to use for beginners, but has plenty to offer advanced users if you're looking to expand your knowledge. There are lots of examples online of people using it for time-lapse, slow-motion, and other video cleverness. You can also use the libraries we bundle with the camera to create effects. The camera works with all models of Raspberry Pi 1, 2, 3 and 4. It can be accessed through the MMAL and V4L APIs, and there are numerous third-party libraries built for it, including the Pi camera Python library.

Cameras are sensitive to static. Earth yourself prior to handling the PCB. A sink tap or similar should suffice if you don't have an earthing strap. The camera board attaches to the Raspberry $\mathrm{Pi}$ via a 15-way ribbon cable. There are only two connections to make: the ribbon cable needs to be attached to the camera PCB, and to the Raspberry Pi itself. You need to get the cable the right way round, or the camera will not work. On the camera PCB, the blue backing on the cable should face away from the PCB, and on the Raspberry Pi it should face towards the Ethernet connection (or where the Ethernet connector would be if you're using a model A).

Although the connectors on the PCB and the $\mathrm{Pi}$ are different, they work in a similar way. On the Raspberry Pi itself, pull up the tabs on each end of the connector. It should slide up easily, and be able to pivot around slightly. Fully insert the ribbon cable into the slot, ensuring it is set straight, then gently press down the tabs to clip it into place. The camera PCB connector also requires you to pull the tabs away from the board, gently insert the cable, then push the tabs back. The PCB connector can be a little more awkward than the one on the Pi itself.[16]

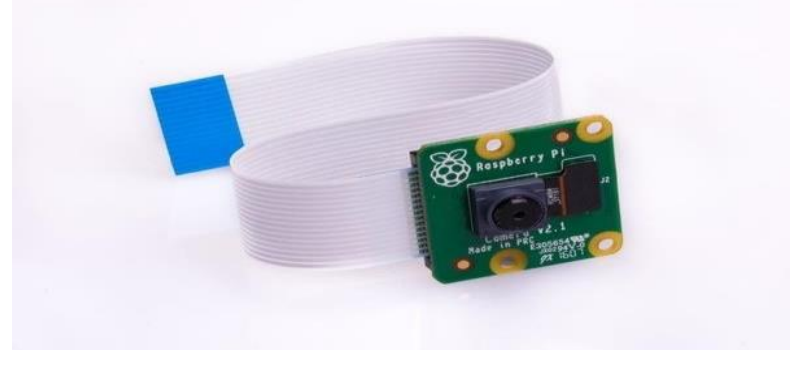

Fig 3: Rasp pi camera

\section{Artificial Intelligence}

Artificial Intelligence (AI) has grown dramatically and becomes more and more institutionalized in the 21 st Century. In this era of interdisciplinary science, of computer science, cybernetics, automation, mathematical logic, and linguistics [6], questions have been raised about the specific concept of AI [7]. Actually, as early as the 1940s and 1950s, scientists in the field of Mathematics, Engineering, and Computer Science had explored the possibilities of artificial 
brains and were trying to define the intelligence of the machine

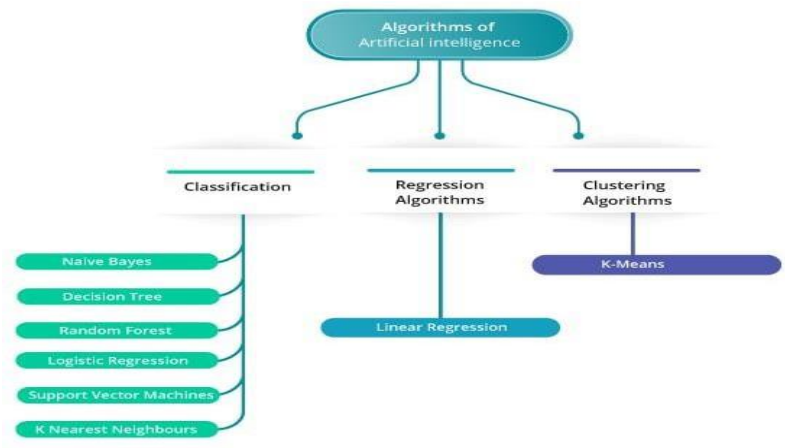

. Fig 4: Artificial Intelligence Algorithm

In 1950, Turing[8] presented the famous "Turing Test" which defined of the concept of "Machine Intelligence". On this background, the origins of AI can be traced to the workshop held on the campus of Dartmouth College in 1965 [9], in which McCarthy persuaded participants to accept the concept of "Artificial Intelligence". It is likewise the beginning of the first "Golden age" of AI. In simple terms, AI aims to extend and augment the capacity and efficiency of mankind in tasks of remaking nature and governing the society through intelligent machines, with the final goal of realizing a society where people and machines coexist harmoniously together [10]. Due to the historical development, AI has been utilized into several major subjects including computer vision, natural language processing, the science of cognition and reasoning, robotics, game theory, and machine learning since the 1980s [11], [12]. These subjects developed independently of each other. However, these disciplines basically had already abandoned the logical reasoning and heuristic. search-based methods which were proposed 30 years ago. Instead, most of them were based on statistical methods which include modelling and learning.

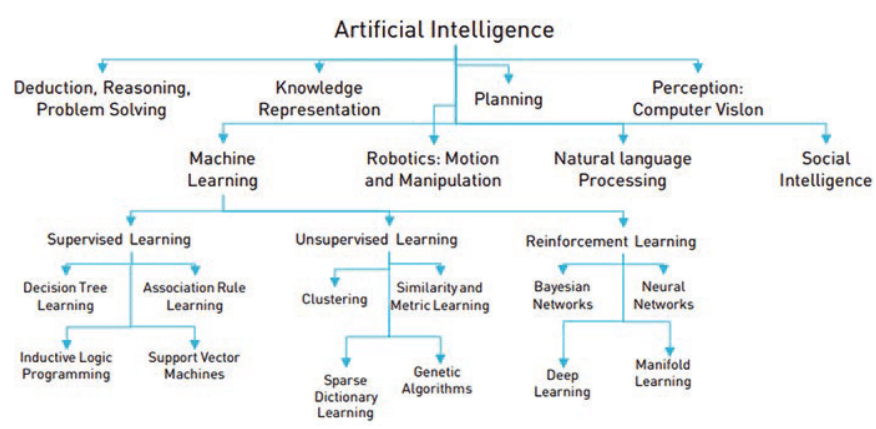

Fig 5: Categorisation of Artificial intelligence

\section{Machine Learning}

Machine learning has evolved from the study of computational learning theory and pattern recognition. It is the most effective method used in the field of data analytics in order to predict something by devising some models and algorithms. These analytics models allows researchers engineers, data scientists and analyst to produce reliable and valid results and decisions. It also helps to discover some hidden patterns or features through historical learning's and trends in data [1]. Feature selection is the most important task of machine learning [3]. Model is created based on the results gathered from the training data that is why machine-learning algorithms are non- interactive. It studies the past observations to make precise predictions. It is a very difficult task to make an accurate prediction rule based on which algorithm can be developed [2]

For example, spammed and non-spammed emails have to be distinguished using machine learning, then this could be done by collecting examples of spammed and non-spammed emails. Then these example are fed into machine-learning algorithm to indicate whether the mails are spammed or not by generating an accurate prediction rule [2]. ML is suitable for dealing with those problems where theoretical knowledge is still insufficient but for which we have an ideal number of observations and results [4].

Machine learning approaches are traditionally divided into three broad categories, depending on the 
nature of the "signal" or "feedback" available to the learning system:

Supervised learning: The computer is presented with example inputs and their desired outputs, given by a "teacher", and the goal is to learn a general rule that maps inputs to outputs.

Unsupervised learning: No labels are given to the learning algorithm, leaving it on its own to find structure in its input. Unsupervised learning can be a goal in itself (discovering hidden patterns in data) or a means towards an end (feature learning).

Reinforcement learning: A computer program interacts with a dynamic environment in which it must perform a certain goal (such as driving a vehicle or playing a game against an opponent). As it navigates its problem space, the program is provided feedback that's analogous to rewards, which it tries to maximize. ${ }^{[3]}$

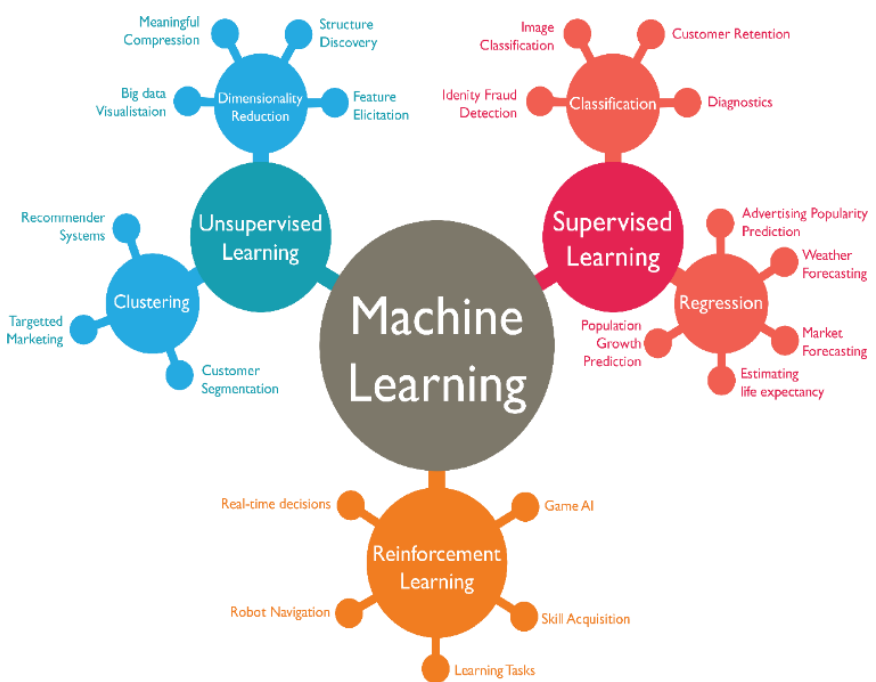

Fig 6: Machine learning Algorithm

Other approaches have been developed which don't fit neatly into this three-fold categorisation, and sometimes more than one is used by the same machine learning system. For example topic modeling, dimensionality reduction or meta learning. ${ }^{[7]}$

As of 2020, deep learning has become the dominant approach for much on going work in the field of machine learning [14]

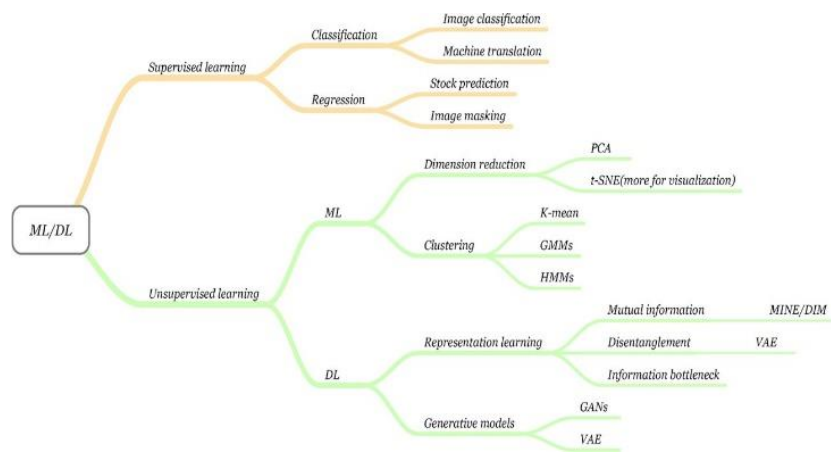

Fig 7: Basic Machine learning with python code.

\section{E. Image Processing}

Image processing is a method to perform some operations on an image, in order to get an enhanced image or to extract some useful information from it. It is a type of signal processing in which input is an image and output may be image or characteristics/features associated with that image. Nowadays, image processing is among rapidly growing technologies. It forms core research area within engineering and computer science disciplines too.

Image processing basically includes the following three steps:

- Importing the image via image acquisition tools;

- Analysing and manipulating the image;

- Output in which result can be altered image or report that is based on image analysis.

There are two types of methods used for image processing namely, analogue and digital image processing. Analogue image processing can be used for the hard copies like printouts and photographs. Image analysts use various fundamentals of interpretation while using these visual techniques. Digital image processing techniques help in manipulation of the digital images by using computers. The three general phases that all types of data have to undergo while using digital technique 
are pre-processing, enhancement, and display, information extraction.[5]

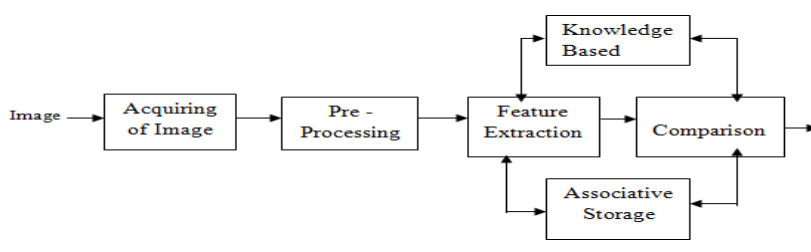

Fig 8: Image processing

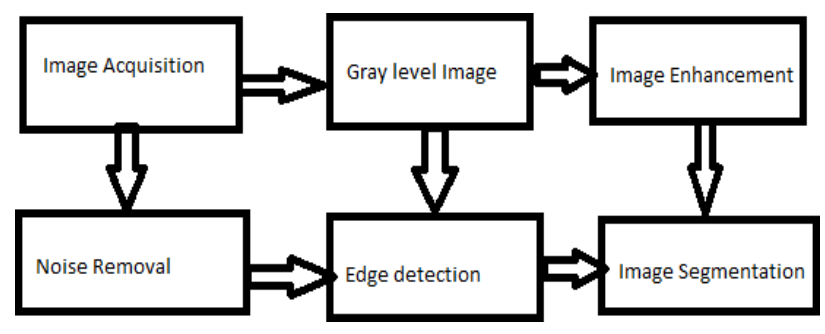

Fig 9: Image processing methodology

\section{F. Python is used for programming}

Python is an interpreted, high-level and generalpurpose programming language. Python's design philosophy emphasizes code readability with its notable use of significant whitespace. Its language constructs and object-oriented approach aim to help programmers write clear, logical code for small and large-scale projects.[17]

Python is dynamically typed and garbage-collected. It supports multiple programming paradigms, including structured (particularly, procedural), object-oriented and functional programming. Python is often described as a "batteries included" language due to its comprehensive standard library.[18]

Python was created in the late 1980s, and first released in 1991, by Guido van Rossum as a successor to the $\mathrm{ABC}$ programming language. Python 2.0, released in 2000, introduced new features, such as list comprehensions, and a garbage collection system with reference counting, and was discontinued with version 2.7 in 2020. Python 3.0, released in 2008, was a major revision of the language that is not completely backward-compatible and much Python 2 code does not run unmodified on Python 3.[21] With Python 2's end-of-life, only

Python 3.6.x and later are supported, with older versions still supporting e.g. Windows 7 (and old installers not restricted to 64-bit Windows).[19]

Python interpreters are supported for mainstream operating systems and available for a few more (and in the past supported many more). A global community of programmers develops and maintains CPython, a free and open-source reference implementation. A non-profit organization, the Python Software Foundation, manages and directs resources for Python and CPython development.[20]

As of December 2020 Python ranked third in TIOBE's index of most popular programming languages, behind $\mathrm{C}$ and Java.[21]

\section{IMPLEMENTATION}

The device we are discussing in this paper will be requiring the Raspberry Pi which will help get the required output. Basically, the device will calculate the lengthy equations with errorless output. The first step will be scanning the equation to be solved, once the system detects the question, it will be displayed and the question will be read out loud for the user to check whether the scanned question is correct or mistaken. To scan the question the device will be using the process called "Image Processing". The standard process of Image processing will be followed by the system to get the enhanced image and extract the exact information from it. Later the system will used the Artificial Intelligence and Machine Learning to get the calculation part done and give the solution. Later, when the equation is solved, the answer will be displayed as well as read out by the device. The flow process of the device is as follows: 


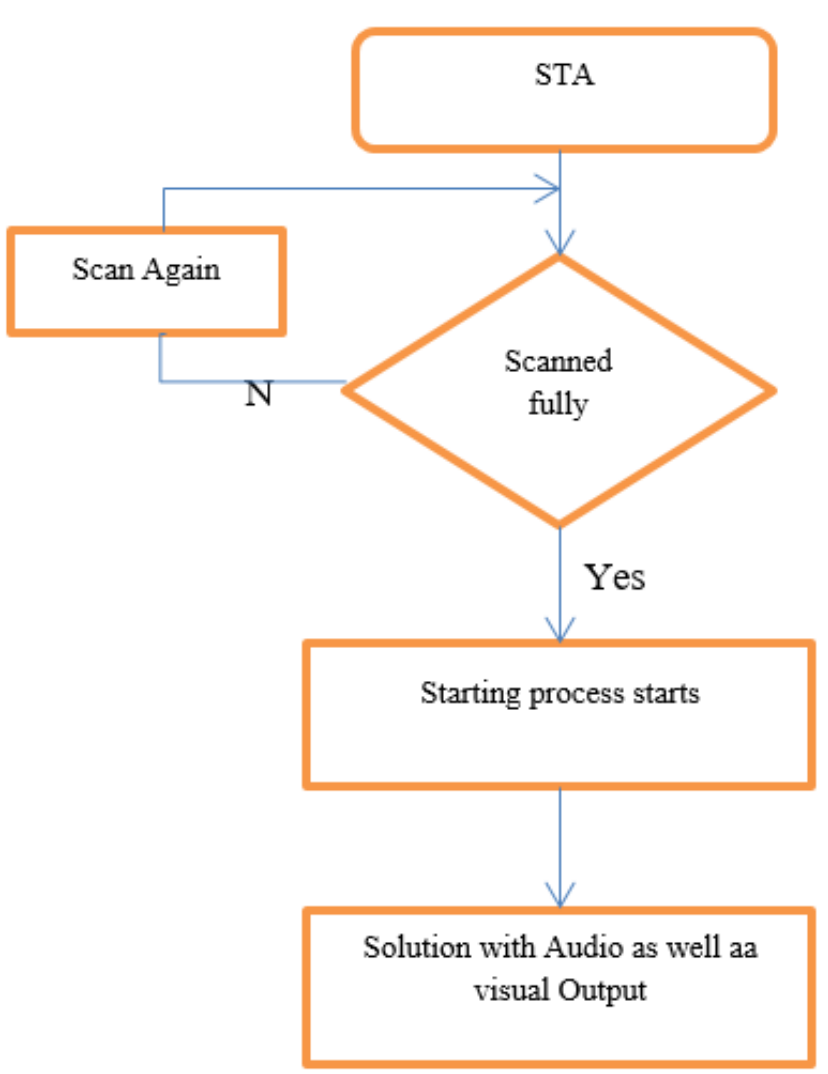

Fig 10 : Flowchart of the process

The diagrammatical representation of the process of proposed idea is as followed:

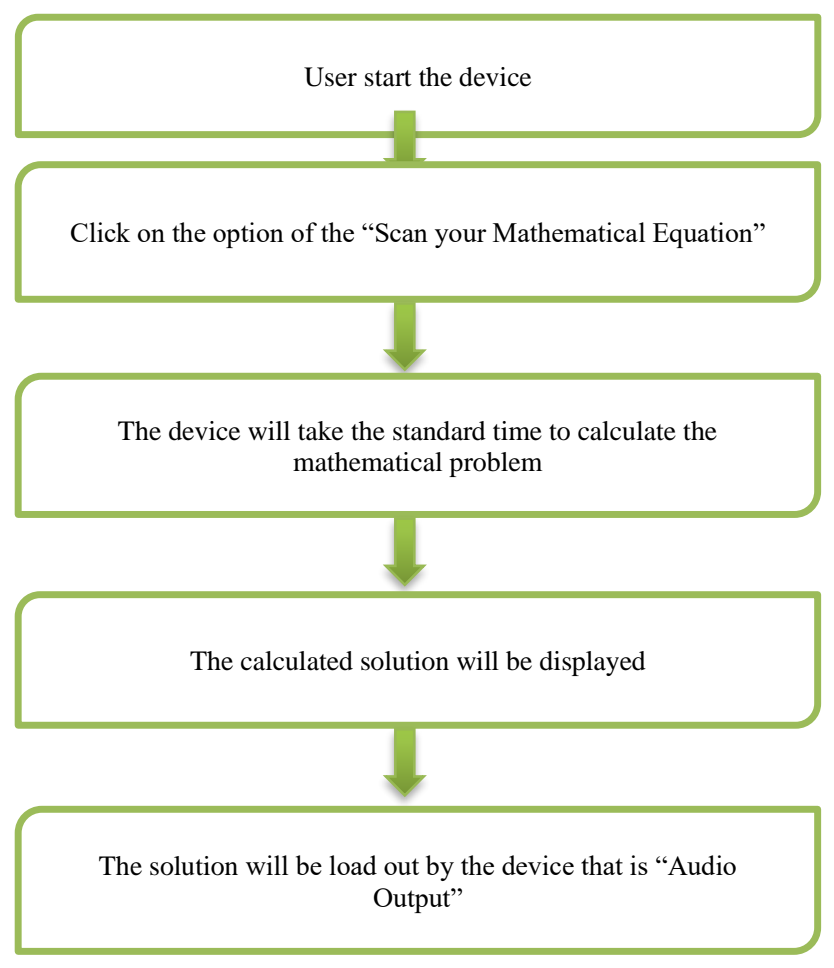

\section{Block Diagram:}

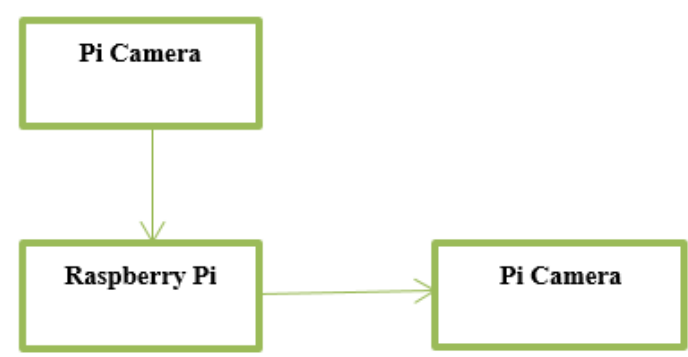

Block diagram of the Presented device.

\section{CONCLUSION \& FUTURE WORK}

In this paper, we have discussed about the device which will calculate the Lengthy and Higher Mathematical Equations. The device will give us the errorless and accurate answer of the inputted equations. This device can solve higher mathematical equations like Fourier Transform, Higher order Matrix, Numerical Equations, Range Kutta Method Equations, etc. this equations are required and also serve as the base of various algorithm which is helping technology to grow. The device will not only ease the process of calculating but also save the precious time. It will convert the complicated process into a simpler one.

The above proposed system will be having a hardware which will look like an advanced calculator or a simple mobile with the simple components as mentioned above. After the successful completion of this project the future scope may include making an application which will be operating as above proposed model. The application will be supported for all types of operating systems. With one click, the application will be useful for solving the higher mathematical problems.

Later, for further future scope, this concept can be installed in the robots who will be available to solve higher mathematical problem by just calling them just like normal robots who carry out the activities being allotted. This has vast role in the field of engineering as the problems faced by common people will be solved. 


\section{REFERENCES}

[1]. "Machine learning: What it is, Why it matters" www.sas.com . Retrieved 2016-09-25

[2]. Schapire, R. E. (2003). The boosting approach to machine learning: An overview. In nonlinear estimation and classification. Springer New York, 149-171.

[3]. Min, F., Hu, Q., \& Zhu, W. (2014). Feature selection with test cost constraint. International Journal of Approximate Reasoning, 55(1), 169-179.

[4]. Lary, D.J., Alavi, A. H., Gandomi, A. H., \& walker, A. L. (2016). Machine learning in geosciences and remote sensing. Geoscience Frontiers, 7(1), 3-10.

[5]. Digital image processing by University of Tartu. www.sisu.ut.ee/imageprocessing.

[6]. D. Vernon, G. Metta, and G. Sandini, "A survey of artificial cognitive systems: Implications for the autonomous development of mental capabilities in computational agents," IEEE Trans. Evol. Comput., vol. 11, no. 2, pp. 151-180, Apr. 2007.

[7]. D. Kirsh, "Thinking with external representations," Ai Soc., vol. 25, no. 4, pp. 441454, 2010.

[8]. M. Turing, "Computing machinery and intelligence," Mind, vol. 59, no. 236, pp. 433- 460, Oct. 1950

[9]. P. McCorduck, Machines Who Think. Natick, MA, USA: A. K. Peters, 2004.

[10].T. J. M. Bench-Capon and P. E. Dunne, "Argumentation in artificial intelligence," Artif. Intell., vol. 171, nos. 10-15, pp. 619-641, 2007.

[11].D. Marr, "Artificial intelligence-A personal view," Artif. Intell., vol. 9, no. 1, pp. 37- 48, 1977.

[12]. N. J. Nilsson, Principles of Artificial Intelligence. San Mateo, CA, USA: Morgan Kaufmann, 2014.

[13]. Bengio, Yoshua; LeCun, Yann; Hinton, Geoffrey (2015). "Deep Learning". Nature. 521(7553): 436-

[14]. 444.Bibcode:2015Natur.521..436L. doi:10.1038/nature14539. PMID 26017442. S2CID 3074096.

[15].Pavel Brazdil, Christophe Giraud Carrier, Carlos Soares, Ricardo Vilalta (2009). Metalearning:
Applications to Data Mining (Fourth ed.). Springer Science+Business Media. pp. 10-14, passim. ISBN 978-3540732624.

[16].https://www.raspberrypi.org/documentation/hard ware/camera

[17].https://www.raspberrypi.org/documentation/config uration/camera.md

[18].Kuhlman, Dave. "A Python Book: Beginning Python, Advanced Python, and Python Exercises". Section 1.1. Archived from the original (PDF) on 23 June 2012.

[19]. "About Python". Python Software Foundation. Retrieved 24 April 2012., second section "Fans of Python use the phrase "batteries included" to describe the standard library, which covers everything from asynchronous processing to zip files."

[20].Peterson, Benjamin (20 April 2020). "Python Insider: Python 2.7.18, the last release of Python 2". Python Insider. Retrieved 27 April 2020.

[21]. "Python Developer's Guide - Python Developer's Guide". devguide.python.org. Retrieved 17 December 2019.

[22]. "History and License". Retrieved 5 December 2016. "All Python Releases are Open Source"

[23]. TIOBE index (December 2020). "TIOBE Index for December 2020". TIOBE.com. Retrieved 20 December 2020.

\section{Cite this article as :}

Prof. Sonali Zunke, Sandesh Ukey, Dipak Mendhe, "Review on Robotic Machine to Solve the Matrix with Natural Language Processing and Image Processing", International Journal of Scientific Research in Computer Science, Engineering and Information Technology (IJSRCSEIT), ISSN : 2456-3307, Volume 7 Issue 3, pp. 395-402, May-June 2021. Available at doi : https://doi.org/10.32628/CSEIT217382 Journal URL : https://ijsrcseit.com/CSEIT217382 